\title{
Short-Term Use of Uncovered Self-Expanding Metallic Airway Stents for Severe Expiratory Central Airway Collapse
}

\author{
Adnan Majid ${ }^{a} \quad$ Daniel Alape $^{a} \quad$ Fayez Kheir $^{b} \quad$ Erik Folch $^{a}$ Sebastian Ochoa ${ }^{a}$ \\ Alejandro Folch $^{\mathrm{a}}$ Sidhu P. Gangadharan ${ }^{\mathrm{a}}$ \\ a Division of Thoracic Surgery and Interventional Pulmonology, Beth Israel Deaconess Medical Center, Harvard \\ Medical School, Boston, MA, and ${ }^{b}$ Division of Pulmonary Diseases, Critical Care and Environmental Medicine, \\ Tulane University Health Sciences Center, New Orleans, LA, USA
}

\section{Keywords}

Airway stabilization · Bronchoscopy · Stent placement ·

Central dynamic airway collapse

\begin{abstract}
Background: Patients with severe symptomatic expiratory central airway collapse (ECAC) undergo a stent trial to determine whether they are candidate for tracheobronchoplasty. Most stent trials were done using silicone stents. However, there was a higher number of silicone stent-related complications. Objectives: The aim of this study was to evaluate the safety and efficacy of short-term uncovered self-expanding metallic airway stents (USEMAS) in patients with ECAC. Methods: This was a retrospective review. Baseline measurements were compared to those obtained after 7-14 days. Measurements included: Modified Medical Research Council (mMRC), Cough Quality of Life Questionnaire (CQLQ), spirometry testing, and 6-Minute Walk Test (6MWT). Stent- and procedure-related complications were reported. Results: 33 patients (median age, 52 years) underwent the USEMAS trial. Presenting symptoms were dyspnea in $100 \%$, intractable cough in $90.3 \%$, recurrent infection in $42.2 \%$, and inability to clear secretions in $21.4 \%$. Dyspnea, cough, and secretion
\end{abstract}

\section{KARGER}

E-Mail karger@karger.com www.karger.com/res clearance improved in 88,70 , and $57 \%$, respectively. Overall, there was a significant improvement in $\operatorname{mMRC}(p<0.001)$, CQLQ $(p=0.015)$, and 6MWT ( $p=0.015)$. There was 1 airway infection, 1 stent migration, and 1 pneumothorax. The median duration of USEMAS was 7 days. All stents were removed without any complications. At the time of stent removal, no granulation tissue was observed in 30.9\%, and mild granulation tissue was observed in $69.1 \%$. Conclusion: The short-term USEMAS trial improves respiratory symptoms, quality of life, and exercise capacity with few complications in patients with severe symptomatic ECAC when performed by a multidisciplinary airway team in highly specialized centers with experience in the evaluation and treatment of this patient population.

(c) 2016 S. Karger AG, Basel

\section{Introduction}

Tracheobronchomalacia (TBM) and excessive dynamic airway collapse (EDAC) are conditions that affect the central airways and frequently cause dyspnea, cough, and recurrent respiratory infections. TBM is characterized by softening of the tracheobronchial cartilaginous 
structures, while EDAC is described as atrophy of the muscular fibers in the posterior membranous wall [1-3]. Although the pathophysiology of airway mechanics in TBM and EDAC is different, the clinical characteristics and therapeutic approach are similar [4-6]. Both conditions are underdiagnosed, since the symptoms are nonspecific and the reported prevalence ranges from 4.1 to $23.3 \%$ [7-11].

We will use the term expiratory central airway collapse (ECAC) to include both patient populations. ECAC commonly occurs in patients with chronic airway inflammation [12]. ECAC is usually diagnosed by dynamic flexible bronchoscopy and quantified by estimating percentage of collapse (measured as anteroposterior distance) or by estimating the collapsibility index on a dynamic airway computed tomography (DACT) [13]. Based on DACT data from healthy volunteers, we classify the degree of ECAC into mild (70-79\%), moderate (80-89\%), and severe $(>90 \%)[14,15]$.

Treatment of symptomatic severe ECAC focuses on airway stabilization. Pneumatic splinting with continuous positive airway pressure (CPAP) has been used with variable success [16]. It requires an external device difficult to use with daily activities, and the long-term effects are unknown [16-19]. In addition, CPAP is usually regarded as a "bridge" to more definitive surgical intervention [3].

Surgical stabilization of the airway with tracheobronchoplasty (TBP) is the definitive treatment for patients with severe symptomatic ECAC and has been shown to improve dyspnea, quality of life, and exercise capacity in carefully selected adults with ECAC $[4,5,20]$. However, before attempting any surgical intervention, symptomatic ECAC patients should undergo evaluation with a stent trial. Silicone stent placement has been used for patients with severe symptomatic ECAC and has been shown to improve respiratory symptoms, quality of life, and functional status $[4,5,13,17-19,21]$. However, silicone stents were associated with a very high complication rate such as stent migration, infection, and mucous plugging [21]. In addition, when using Y-shaped stents, a subset of patients may develop severe intractable cough and thus limiting the interpretation of the trial.

Uncovered self-expanding metallic airway stents (USEMAS) is an alternative technique that has been used mainly for palliation in malignant airway obstruction [22]. Long-term use of USEMAS in benign airway diseases is controversial and not recommended [23]. Recently, an abstract and letter to the editor from our group reported the outcomes of few cases of short-term USEMAS use in severe symptomatic ECAC [24, 25].
The aim of this study was to assess the efficacy and safety of short-term USEMAS use in improving symptoms, quality of life, and exercise capacity in patients with severe symptomatic ECAC who are candidates for TBP.

\section{Material and Methods}

\section{Study Design}

We conducted a retrospective review from our prospective database of patients with severe ECAC between February 2013 until December 2015. The study protocol was approved by the Beth Israel Deaconess Medical Center Institutional Review Board, Boston, MA (IRB No. 2014P-000013).

\section{Subject Population and Study Outcome}

The diagnosis of ECAC was made by the combination of DACT imaging and bronchoscopy. Severe ECAC was defined as a collapse $>90 \%$ of the airway during exhalation [7]. All patients with severe symptomatic ECAC who failed to respond to maximal medical therapy and underwent USEMAS placement in the central airways (tracheal, main stem bronchus/bronchus intermedius, or both) were included in the study.

Initial treatment of symptomatic ECAC includes supportive treatment of recurrent infections, aggressive therapy for concomitant diseases, such as asthma or chronic obstructive pulmonary disease, pulmonary physiotherapy, CPAP for obstructive sleep apnea, and gastroesophageal reflux. Furthermore, patients are provided with airway clearance techniques such airway oscillatory devices (flutter valve) or external percussion vests to minimize mucostasis as well as educated about breathing techniques such as pursed lip breathing. Following optimization of all coexisting conditions, a functional assessment is performed [e.g., PFTs, 6-Minute Walk Test (6MWT), quality of life assessment, dyspnea, and cough scores] in patients who remain symptomatic.

Age, gender, comorbidities, type, severity, and location of ECAC (diffuse vs. local) and patients' self-reported respiratory symptoms were recorded at baseline. All patients underwent DACT and dynamic bronchoscopic assessment. Patient-reported measures included the Cough Specific Quality of Life Questionnaire (CQLQ) [26] and modified Medical Research Council (mMRC) dyspnea scale [27]. In addition, patients underwent physiologic and functional assessment (spirometry testing and 6MWT).

All patients underwent a dynamic flexible bronchoscopy as per protocol, and if severe central dynamic airway collapse was documented, we proceeded with a rigid bronchoscopy and USEMAS placement. Procedure- and stent-related complications were recorded. All patients who underwent stent trial were on a stent maintenance protocol [using $10 \mathrm{~mL}$ normal saline solution for 10 min b.i.d., guaifenesin 600-1,200 mg p.o. b.i.d. and Flutter valve (10 times) b.i.d.] [28]

At a scheduled follow-up visit no more than 7 days after the procedure, patients were asked whether symptoms had improved and baseline measurements were repeated. All patients were scheduled for a rigid bronchoscopy and stent removal 10-14 days after initial placement. 
Fig. 1. Dynamic CT scan in the expiratory phase before and during stent trial. a Tracheobronchomalacia with near complete flattening of the trachea. b Intact well-positioned USEMAS in the distal trachea keeping the airway open. c Coronal view of the airway with USEMAS situated in the trachea and left main bronchus.

Fig. 2. Dynamic bronchoscopy and stent trial. a Distal trachea during inspiration. b Distal trachea during exhalation. c Stent deployed in the distal trachea, left main bronchus, and right main bronchus. d Mild granulation tissue after stent removal.
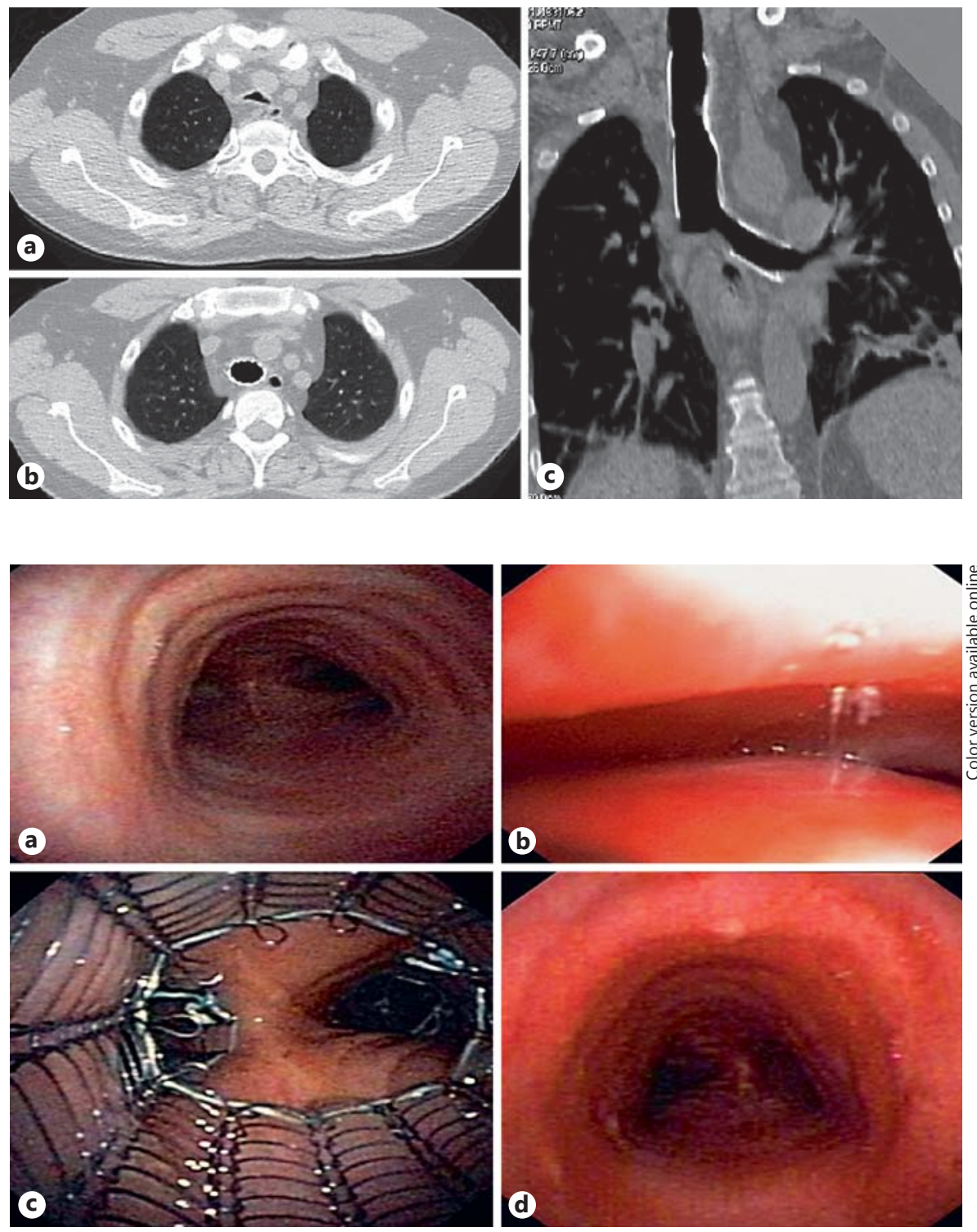

\section{ECAC Evaluation}

Dynamic Bronchoscopy Protocol

Patients with suspected ECAC underwent bronchoscopy under minimal sedation using intravenous midazolam and fentanyl to allow spontaneous respiration. Oral anesthesia was accomplished using 10-20 $\mathrm{mL}$ of $1 \%$ atomized lidocaine to suppress gag reflex and $1 \%$ lidocaine in $2-\mathrm{mL}$ aliquots were delivered through the bronchoscope during the procedure to irrigate the vocal cords, aryepiglottic folds, and entire tracheobronchial tree. An Olympus BF P180 video bronchoscope (Olympus America, Melville, NY, USA) with a 4.9-mm outer diameter and $2.0-\mathrm{mm}$ working channel was used to minimize any stenting effect. The bronchoscope was introduced into the proximal trachea at the level of the cricoid. At that point, patients were instructed to take a deep breath, hold it and then blow it out (forced expiratory maneuver). Maneuver was done at the following 6 sites: proximal trachea at the level of the cricoid; mid trachea $5 \mathrm{~cm}$ proximal to the carina; distal trachea $2 \mathrm{~cm}$ proximal to the carina; right main stem bronchus at the right tracheobronchial angle; bronchus intermedius at the level of the right upper lobe bronchus, and left main bronchus at the left tracheobronchial angle. The maneuver was repeated three times to ensure maximal airway narrowing during exhalation.

\section{CT Central Airway Protocol}

All patients were imaged according to our standard low dose CT central airway protocol $[15,29]$, which included imaging during end-inspiratory and continuous dynamic expiratory phases (Fig. 1). A 64-detector row scanner (LightSpeed; GE Medical Systems, Milwaukee, WI, USA, or Aquilion; Toshiba America Medical Systems, Tustin, CA, USA) was used. Helical scanning was performed in the craniocaudal dimension during full inhalation and forced expiration. For both sequences, images were transferred to 
Table 1. Demographics and baseline characteristics

\begin{tabular}{|c|c|c|c|c|}
\hline Demographics & $\begin{array}{l}\text { Total } \\
(n=33)\end{array}$ & $\begin{array}{l}\text { TBM group } \\
(n=21)\end{array}$ & $\begin{array}{l}\text { EDAC group } \\
(n=12)\end{array}$ & $p$ value \\
\hline Median age (IQR), years & $52(44-64)$ & $52(44-65)$ & $53(42-62)$ & 0.207 \\
\hline Median BMI (IQR) & $34.5(30.2-39.2)$ & $34.6(33-40)$ & $30(22.6-37.7)$ & 0.45 \\
\hline Sex & & & & 0.443 \\
\hline Female & $25(75.8)$ & $6(28.6)$ & $2(16.7)$ & \\
\hline Male & $8(24.2)$ & $15(71.4)$ & $10(83.3)$ & \\
\hline Smoking history & & & & 0.125 \\
\hline No & $22(66.7)$ & $12(57.1)$ & $10(83.3)$ & \\
\hline Yes & $11(33.3)$ & $9(42.9)$ & $2(16.7)$ & \\
\hline Symptoms prior to stent trial & & & & 0.641 \\
\hline Shortness of breath & $33(100)$ & $21(100)$ & $12(100)$ & \\
\hline Cough & $30(90.9)$ & $19(90.5)$ & $11(91.7)$ & \\
\hline Recurrent infection & $14(42.2)$ & $10(47.6)$ & $4(33.3)$ & \\
\hline Inability to clear secretions & $7(21.2)$ & $6(28.6)$ & $1(8.3)$ & \\
\hline
\end{tabular}

Data given as $n(\%)$ unless otherwise specified. IQR, interquartile range; GERD, gastroesophageal reflux disease; COPD, chronic obstructive disease; OSA, obstructive sleep apnea; PVCM, paradoxical vocal cord motion.

a picture archiving communication system (Centricity, version 3.1; GE Medical Systems) and interpreted by an experienced thoracic radiologist. To evaluate the cross-sectional area of the airway (square millimeters), the airway luminal border was manually traced on both end-inspiratory and forced-expiratory images. The percentage of luminal collapse between end inspiration and forced expiration was calculated using the following formula: $\mathrm{C}_{\mathrm{LU}}=[1-$ $\left.\left(A_{e e} / A_{e i}\right)\right], \times 100$, where $C_{L U}$ is luminal collapse, $A_{e e}$ is luminal area at end expiration, and $\mathrm{A}_{\mathrm{ei}}$ is luminal area at end inspiration.

\section{Operative Technique}

All airway stents (Fig. 2) were placed in the operating room with the patient under total intravenous anesthesia. Following anesthesia induction, a rigid bronchoscope (Bryan-Dumon Series II; Bryan Corporation; Woburn, MA, USA) was introduced, and respiration was maintained through jet ventilation. After a complete airway inspection, the length and diameter of the trachea, left and right main stem bronchi, and bronchus intermedius were measured. The anterior posterior and lateral diameters of the airway were estimated using a tracheobronchial stent sizing device (Aerosizer ${ }^{\mathrm{TM}}$; Merit Medical Endotek, South Jordan, UT, USA) and the coronal, axial, and sagittal views at the end of inspiration on DACT. We tend to oversize the stent diameter by $1 \mathrm{~mm}$ from airway size of interest as measured on CT scan and tracheobronchial stent sizing device during the procedure. We placed either one or multiple self-expanding uncovered metallic stents (Ultraflex ${ }^{\mathrm{TM}}$ Tracheobronchial Stent System; Boston Scientific Corporation, Natick, MA, USA) under direct bronchoscopic guidance and a good fit was confirmed visually. All stents were dilated using a CRE ${ }^{\mathrm{TM}}$ dilating balloon (Boston Scientific, Natick, MA, USA) up to the predetermined size to minimize the risk of postoperative migration.

The patients were scheduled in the next 10-14 days for stent removal in the operating room. The airway was assessed for granulation tissue, stent migration, device fracture, mucus plugging, and infection.
Granulation tissue formation was graded on a 4-point scale: 0 , no granulation tissue; 1 , mild (less than $25 \%$ ) obstruction; 2 , moderate (25-50\%) obstruction, and 3 , severe (above $50 \%$ ) obstruction [30]. Procedure time was recorded and stent removal process was rated easy or difficult by the operator.

\section{Statistical Methods}

The efficacy of USEMAS airway stabilization was assessed by comparing self-reported respiratory symptoms, patient-reported measures (mMRC and CQLQ), and functional assessments (spirometry and $6 \mathrm{MWT}$ ) following intervention with baseline measures. Comparisons were performed on a per-patient basis, with the net change between baseline and postintervention measures as the unit of analysis. Statistical analysis was performed using SPSS version 21.0 for Macintosh (International Business Machines Corp., Armonk, NY, USA), with a $p$ value of $<0.05$ defined as significant. Descriptive statistics including median, range, and percentage were utilized to describe patient demographics and outcomes. The baseline and follow-up measurements were compared with Wilcoxon signed-rank test.

\section{Results}

Thirty-three patients with severe symptomatic ECAC underwent USEMAS placement (Table 1). Patients had a median age of 52 years [interquartile range (IQR) 44-64] and $75.8 \%(n=25)$ were female. The baseline demographics and symptoms of the patients are shown in Table 1. Dynamic CT scan and bronchoscopy showed that 21 patients had TBM and 12 patients had EDAC. A total of $21(63.3 \%)$ patients had gastroesophageal reflux dis- 
Table 2. Clinical outcomes following self-expanding metallic stent placement trial in the whole population

\begin{tabular}{|c|c|c|c|c|c|}
\hline Outcome measures & Baseline & During stent placement & Change & Improved, $n /$ total & $p$ value \\
\hline $\operatorname{mMRC}(n=32)$ & $3(2-3)$ & $1(1-3)$ & -2 & $21 / 31$ & $<0.001$ \\
\hline $\operatorname{CQLQ}(n=25)$ & $73(62-80)$ & $64(49-75)$ & -9 & $16 / 25$ & 0.015 \\
\hline 6MWT ft. $(n=23)$ & $1,135(900-1,502)$ & $1,342(1,003-1,597)$ & 207 & $19 / 23$ & 0.015 \\
\hline FEV1 L $(n=27)$ & $2.15(1.55-2.61)$ & $1.99(1.42-2.72)$ & -0.16 & $16 / 27$ & 0.304 \\
\hline
\end{tabular}

Data are presented as median (IQR). IQR, interquartile range; CQLQ, Cough Specific Quality of Life Questionnaire; mMRC, modified Medical Research Council dyspnea scale; 6MWT, 6-Minute Walk Test; FEV1, forced expiratory volume during the first second.

Table 3. Symptoms during stent trial in the whole population

\begin{tabular}{llll}
\hline Symptoms & Improved & Got worse & Unchanged \\
\hline Dyspnea $(n=33)$ & $29(88)$ & $1(3)$ & $3(9)$ \\
Cough $(n=30)$ & $21(70)$ & $6(20)$ & $3(10)$ \\
Ability to clear secretion $(n=7)$ & $4(57)$ & $1(14.3)$ & $2(28.7)$ \\
\hline \multicolumn{2}{c}{ Data given as $n(\%)}$. & & \\
\hline
\end{tabular}

ease, $13(45.5 \%)$ had asthma, $12(36.4 \%)$ had paradoxical vocal cord motion, $12(36.4 \%)$ had obstructive sleep apnea, and $4(12.1 \%)$ had chronic obstructive pulmonary disease.

Dyspnea $(100 \%, n=33)$, intractable coughing (90.9\%, $n=30)$, recurrent infection $(42.4 \%, n=14)$, and inability to clear secretion $(21.2 \%, n=7)$ were the presenting symptoms. Thirty-one patients had diffuse ECAC and 2 patients had localized ECAC: 1 in the left main bronchus and the other in the cervical trachea. The median time of the procedure (dynamic flexible bronchoscopy, rigid bronchoscopy, and stent placement) was $51 \mathrm{~min}$ (IRQ 41-63). Overall, 84 USEMAS were deployed under rigid bronchoscopy and general anesthesia. Three patients had 1 stent, 13 patients 2 stents, 13 patients 3 stents, and 4 patients 4 stents. The stents were distributed as follow: 28 in the trachea (size range 12-20 $\times 40-60 \mathrm{~mm}$ ), 30 in the left main bronchi (size range $12-16 \times 30-40 \mathrm{~mm}$ ), 13 in the right main bronchi (size range $12-16 \times 20-40 \mathrm{~mm}$ ), and 13 in the bronchus intermedius (size range $10-14 \times-20$ $40 \mathrm{~mm}$ ).

\section{Overall}

There was a significant improvement in mMRC $(p<$ $0.001)$, CQLQ $(p=0.015)$, and 6MWT $(p=0.015)$, respectively. There was no significant change in FEV1 (Table 2). On self-reported respiratory symptoms, dyspnea improved in $29(88 \%)$, cough improved in 21 (70\%), and ability to clear secretions improved in 4 (57\%) patients, respectively (Table 3).

Procedure-related complications included: sore throat in $9(27.2 \%)$ patients, laryngospasm in $2(6.1 \%)$, and reversible transient desaturation $\left(<90 \% \mathrm{SpO}_{2}\right)$ in 4 $(12.2 \%)$. In terms of stent-related complications, there was 1 airway infection, 1 stent migration, and 1 pneumothorax but no mucus plugging or stent fracture. The median duration of USEMAS was 7 days (range 3-21). All stents were removed without any complications and were rated as easy removal by the operator. At the time of stent removal, no granulation tissue was observed in $30.9 \%(n=26 / 84)$ and mild granulation tissue in $69.1 \%$ $(n=58 / 84)$. Chest pain/pressure was present in 11 patients (33.3\%), all of which had more than 3 stent placements, and pain was adequately controlled with analgesics.

A total of 25 (75.6\%) patients had responded positively to the stent trial based on combination of improvement in self-reported respiratory symptoms, patient's objective measurements, and functional status. Thirteen patients underwent TBP, 8 patients are waiting for further optimization of comorbidities (weight loss, Nissen fundoplication for gastroesophageal reflux disease), 2 patients are scheduled for surgery, and 2 patients declined surgery and are currently on medical treatment (expectorant, flutter valve, bronchodilators, CPAP, and pulmonary rehabilitation). 


\section{By Group}

Tracheobronchomalacia

In patients with TBM, there was a significant improvement in mMRC $(p<0.001)$, CQLQ $(p=0.006)$, and $6 \mathrm{MWT}(p=0.025)$, respectively. There was no significant change in FEV1. On self-reported respiratory symptoms, dyspnea improved in 19 (90.5\%), cough improved in 15 (78\%), and ability to clear secretions improved in 4 (66.6\%) patients, respectively.

\section{Excessive Dynamic Airway Collapse}

In patients with EDAC, there was a clinically significant change in mMRC, CQLQ, and 6MWT, but it did not reach statistical significance probably due to the small sample size. There was no significant change in FEV1. On self-reported respiratory symptoms, dyspnea improved in $10(83.3 \%)$ and cough improved in $6(54.5 \%)$ patients, respectively.

\section{Outcome of TBP}

A total of 13 patients ( 11 with TBM and 3 with EDAC) with successful stent trial underwent TBP. At the 3-month follow-up, there was a significant improvement in mMRC, CQLQ, 6MWT, but not in FEV1. On self-reported respiratory symptoms', dyspnea improved in $77 \%(n=10 / 13)$, cough improved in $84.6 \%(n=11 / 13)$, and ability to clear secretions improved in $83.3 \%$ (5/6) patients, respectively.

\section{Discussion}

Although the use of metallic stent in malignant airway obstruction is established, the role of long-term use of metallic stent in benign airway disease is still controversial and not recommended. Given the fact that metallic stents were placed for a long time in patients with benign airway disease, several investigators had reported a number of serious adverse events leading the FDA to publish an advisory that metallic stents should be used as the last resort for patients with benign airway disorders after exploring other options [22]. However, it is important to affirm that our rationale of using USEMAS instead of silicone is (1) the preferable inner diameter to wall thickness ratio, (2) fewer episodes of stent migration, (3) better ability to conform to tortuous airways, (4) less airway obstruction due to secretions, and (5) its short-term use that has not been associated with any serious complications up to our knowledge [31,32]. Also, silicone stents were associated with a high complication rate, occurring usually in the first 4 weeks after placement, and only about $55 \%$ of patients with severe symptomatic ECAC responded favorably to the stent trial $[4-6,21]$.

Alazemi et al. [33] reported the outcome, health care resource utilization (HRU), and costs of 46 patients who had metallic stent removal after a median in situ duration of 292 days. A total of $80 \%$ of metallic stents were placed for benign airway disorder. Although, the removals of metallic stents were feasible, they were associated with significant complications, HRU, and costs. However, there were fewer complications, HRU, hospital stay, and costs when the stents were in the airways for $\leq 30$ days before their removal as compared to $>30$ days [33]. Another study by Chan et al. [34] showed a higher long-term complication rate in patients who had metallic stent for benign airway disease, but $90 \%$ of stents were removed uneventfully. Thus, it is important to emphasize that when metallic stents are left for a prolonged period of time, they are harder to remove and are associated with increased complications [33, 34].

The use of metallic and silicone stents for benign airways has also been described previously in lung transplant patients with malacia and showed improvement in spirometry values [35]. Besides, the short-term ( $<30$ days) complications of metallic stents in transplant patients with airway dehiscence were mucus plugging in $11.5 \%$ and stent migration in $3.3 \%$ of patients [36]. Our study showed that the patients' reported symptoms of dyspnea, cough, and ability to clear secretions improved by 88,70 , and $57 \%$, respectively. This was associated with a significant improvement of the patients' reported measures (mMRC and CQLQ) as well as 6MWT. In addition, 75.6\% of patients responded favorably to stent trial. Importantly, only $3.3 \%(n=1 / 33)$ had infection treated with an oral antibiotic course, $3.3 \%(n=1 / 33)$ had stent migration, and $3.3 \%(n=1 / 33)$ had pneumothorax that was attributed to severe cough after stent removal, but there was no evidence of airway mucosal injury. Eleven patients (33.3\%) had chest pain that was probably due to stent oversizing as part of the natural learning curve by the investigators, leading to excessive radial pressure alongside the airway but could be well controlled with analgesics. The serious complications of USEMAS (defined as stent migration, obstruction, respiratory failure, hemoptysis, pneumothorax) were $6.1 \%$ and compared very favorably with silicone stent complications before (40\%) and after (9\%) adoption of a protocol using mucolytic and expectorant therapy $[21,28]$. Also, the cost of the USEMAS is approximately USD 2,500 per stent (with an average of USD 7,500 per patient) as compared to a Y silicone stent 
(USD 700). Currently, a trial in our center is underway to assess silicone stent efficacy in patients with ECAC.

USEMAS removal was uneventful, and there was no significant granulation tissue formation compared to higher rates reported in other studies $[33,34]$. This is due to the fact that stents were placed for a short time before neo-epithelialization occured. On the other hand, FEV1 was not associated with any significant change. Previous studies have shown that central airway collapse did not correlate with obstruction assessed by FEV1 [37] and was also consistent with previous studies showing clinical improvement after airway stabilization without change in FEV1 $[4,21]$.

This study has several limitations. First, we did not have baseline and follow-up measures for all included patients. In some cases, patients were not able to perform $6 \mathrm{MWT}$ or spirometry test due to their comorbidities and symptoms. In other cases, the admission process to clinic visit was too rapid to allow baseline assessment or patients were unavailable to have follow-up measurements. This could lead to possible bias in favorable outcome. Second, this is an observational trial without a controlled arm. Finally, both patients and clinicians were unblinded to diagnosis and USEMAS trial. Although an objective standard research tool was used to measure outcomes, a potential observer bias cannot be ruled out. It should be emphasized that the insertion of metallic stent in patients with ECAC must be limited to (1) centers that regularly treat such a population in a multidisciplinary fashion, (2) thoracic surgeons experienced in TBP if patients responded clinically to stent trial, and (3) USEMAS inserted for a short term ( $<14$ days) using an implemented protocol.

\section{Conclusion}

Short-term airway stabilization with USEMAS in highly selected patients with severe symptomatic ECAC after optimizing medical therapy might be effective in alleviating symptoms, improving health-related quality of life, as well as exercise capacity when performed by and experienced multidisciplinary airway team and could help identify patients who would benefit from surgical stabilization. Due to high cost and concerns of potential complications, the use of USEMAS in EDAC should be limited to highly specialized centers with experience in the evaluation and treatment of this patient population.

\section{Financial Disclosure and Conflicts of Statement}

A.M. and E.F. are educational consultants for Boston Scientific Corporation. The company did not have any influence in the study design, data analysis, or results presented in the manuscript. The other authors have no potential conflicts of interest or financial support to disclose.

\section{References}

$>1$ Leong P, Bardin PG, Lau KK: What's in a name? Expiratory tracheal narrowing in adults explained. Clin Radiol 2013;68:12681275.

-2 Murgu S, Colt H: Tracheobronchomalacia and excessive dynamic airway collapse. Clin Chest Med 2013;34:527-555.

$\checkmark 3$ Ridge CA, O’Donnell CR, Lee EY, Majid A, Boiselle PM: Tracheobronchomalacia: current concepts and controversies. J Thorac Imaging 2011;26:278-289.

$\checkmark 4$ Majid A, Guerrero J, Gangadharan S, et al: Tracheobronchoplasty for severe tracheobronchomalacia: a prospective outcome analysis. Chest 2008;134:801-807.

5 Ernst A, Odell DD, Michaud G, Majid A, Herth FF, Gangadharan SP: Central airway stabilization for tracheobronchomalacia improves quality of life in patients with chronic obstructive pulmonary disease. Chest 2011; 140:1162-1168.

Short-Term Use of USEMAS for Severe ECAC
6 Gangadharan SP, Bakhos CT, Majid A, Kent MS, Michaud G, Ernst A, Ashiku SK, DeCamp MM: Technical aspects and outcomes of tracheobronchoplasty for severe tracheobronchomalacia. Ann Thorac Surg 2011;91: 1574-1580.

7 Majid A, Fernández L, Fernandez-Bussy S, Herth F, Ernest A: Tracheobronchomalacia (in Spanish). Arch Bronconeumol 2010;46: 196-202.

$>8$ Jokinen K, Palva T, Sutinen S, et al: Acquired tracheobronchomalacia. Ann Clin Res 1977; 9:52-57.

$\checkmark 9$ Ikeda S, Hanawa T, Konishi T, et al: Diagnosis, incidence, clinicopathology and surgical treatment of acquired tracheobronchomalacia (in Japanese). Nihon Kyobu Shikkan Gakkai Zasshi 1992;30:1028-1035.

$\checkmark 10$ Palombini BC, Villanova CA, Araujo E, et al: A pathogenic triad in chronic cough: asthma, postnasal drip syndrome, and gastroesophageal reflux disease. Chest 1999;116:279-284.
1 Jokinen K, Palva T, Nuutinen J: Chronic bronchitis: a bronchologic evaluation. ORL J Otorhinolaryngol Relat Spec 1976;38:178-186.

12 Carden KA, Boiselle PM, Waltz DA, Ernst A: Tracheomalacia and tracheobronchomalacia in children and adults. An in-depth review. Chest 2005;127:984-1005.

13 Lee KS, Sun MR, Ernst A, Feller-Kopman D, Majid A, Boiselle PM: Comparison of dynamic expiratory CT with bronchoscopy for diagnosing airway malacia: a pilot evaluation. Chest 2007;131:758-764.

14 Boiselle PM, O’Donnell CR, Bankier AA, et al: Tracheal collapsibility in healthy volunteers during forced expiration: assessment with multidetector CT. Radiology 2009;252:255262.

15 Litmanovich D, O’Donnell CR, Bankier AA, et al: Bronchial collapsibility at forced expiration in healthy volunteers: assessment with multidetector CT. Radiology 2010;257:560567. 
16 Ferguson GT, Benoist J: Nasal continuous positive airway pressure in the treatment of tracheobronchomalacia. Am Rev Respir Dis 1993;147:457-461.

17 Tamura Y, Sakatani K, Yamakoshi N, et al: A case of severe COPD associated with tracheobronchial stenosis, treated with non-invasive positive pressure ventilation (in Japanese). Nihon Kokyuki Gakkai Zasshi 2008;46:915920.

$>18$ Ferguson GT, Benoist J: Nasal continuous positive airway pressure in the treatment of tracheobronchomalacia. Am Rev Respir Dis 1993; 147:457-461.

19 Adliff M, Ngato D, Keshavjee S, et al: Treatment of diffuse tracheomalacia secondary to relapsing polychondritis with continuous positive airway pressure. Chest 1997;112: 1701-1704.

-20 Odell DD, Shah A, Gangadharan SP, Majid A, Michaud G, Herth F, Ernst A: Airway stenting and tracheobronchoplasty improve respiratory symptoms in Mounier-Kuhn syndrome. Chest 2011;140:867-873.

21 Ernst A, Majid A, Feller-Kopman D, Guerrero J, Boiselle $\mathrm{P}$, Loring $\mathrm{SH}$, O’Donnell C, Decamp M, Herth FJ, Gangadharan S, et al: Airway stabilization with silicone stents for treating adult tracheobronchomalacia: a prospective observational study. Chest 2007;132: 609-616.

22 FDA public health notification: complications from metallic tracheal stents in patients with benign airway disorders. 2005. http://www.fda.gov/MedicalDevices/Safety/ AlertsandNotices/PublicHealthNotifications/ucm062115.htm (accessed July 7, 2015).
23 Jantz M, Silvestri G: Silicone stents versus metal stents for management of benign tracheobronchial disease. Pro: metal stents. J Bronchol 2000;7:177-183

24 Ochoa S, Cheng GZ, Folch E, Majid A: Use of self-expanding metallic airway stents in tracheobronchomalacia. J Bronchology Interv Pulmonol 2015;22:e9-e11.

25 Ochoa S, Folch E, Cheng GZ, Gangadharan S, Majid A: Short-term trial of uncovered selfexpanding metallic airway stents in tracheobronchomalacia. Am J Respir Crit Care Med 2015;191:A3728

26 French C, Irwin R, Fletcher K, Adams T: Evaluation of a cough-specific quality- of-life questionnaire. Chest 2002;121:1123-1131.

27 Bestall JC, Paul EA, Garrod R, Garnham R, Jones PW, Wedzicha JA: Usefulness of the Medical Research Council (MRC) dyspnoea scale as a measure of disability in patients with chronic obstructive pulmonary disease. Tho$\operatorname{rax} 1999 ; 54: 581-586$

28 Odell DD, Majid A, Gangadharan SP, Ernst A: Adoption of a standardized protocol decreases serious complications of airway stenting in patients with tracheobronchomalacia. Chest 2010;138(suppl 4):A784.

29 Zhang J, Hasegawa I, Feller-Kopman D, et al: 2003 AUR Memorial award: dynamic expiratory volumetric CT imaging of the central airways: comparison of standard dose and low dose techniques. Acad Radiol 2003;10:719724 .
30 Shlomi D, Peled N, Shitrit D, Bendayan D, Amital A, Kramer MR: Protective effect of immunosuppression on granulation tissue formation in metallic airway stents. Laryngoscope 2008;118:1383-1388.

-31 Lehman JD, Gordon RL, Kerlan RK Jr, Laberge JM, Wilson MW, Golden JA, Webb WR: Expandable metallic stents in benign tracheobronchial obstruction. J Thorac Imaging 1998;13:105-115.

32 Wahidi MM, Herth FJ, Ernst A: State of the art: interventional pulmonology. Chest 2007; 131:261-274.

33 Alazemi S, Lunn W, Majid A, Berkowitz D, Michaud G, Feller-Kopman D, Herth F, Ernst A: Outcomes, health-care resources use, and costs of endoscopic removal of metallic airway stents. Chest 2010;138:350-356.

-34 Chan AL, Juarez MM, Allen RP, Albertson TE: Do airway metallic stents for benign lesions confer too costly a benefit? BMC Pulm Med 2008;8:7.

35 Chhajed PN, Malouf MA, Tamm M, et al: Interventional bronchoscopy for the management of airway complications following lung transplantation. Chest 2001;120:1894-1899.

-36 Gottlieb J, Fuehner T, Dierich M, Wiesner O, Simon AR, Welte T: Are metallic stents really safe? A long-term analysis in lung transplant recipients. Eur Respir J 2009;34:1417-1422.

37 Loring SH, O’Donnell CR, Feller-Kopman DJ, Ernst A: Central airway mechanics and flow limitation in acquired tracheobronchomalacia. Chest 2007;131:1118-1124. 